\title{
Hybrid Data Fusion and Tracking for Positioning with GNSS and 3GPP-LTE
}

\author{
Christian Mensing, Stephan Sand, and Armin Dammann \\ German Aerospace Center (DLR), Institute of Communications and Navigation, Oberpfaffenhofen, 82234 Wessling, Germany \\ Correspondence should be addressed to Christian Mensing, christian.mensing@dlr.de
}

Received 13 November 2009; Revised 1 April 2010; Accepted 1 June 2010

Academic Editor: Marco Luise

Copyright ( $) 2010$ Christian Mensing et al. This is an open access article distributed under the Creative Commons Attribution License, which permits unrestricted use, distribution, and reproduction in any medium, provided the original work is properly cited.

\begin{abstract}
Global navigation satellite systems (GNSSs) can provide reliable positioning information under optimum conditions, where at least four satellites can be accessed with sufficient quality. In critical situations, for example, urban canyons or indoor, due to blocking of satellites by buildings and severe multipath effects, the GNSS performance can be decreased substantially. To overcome this limitation, we propose to exploit additionally information from communications systems for positioning purposes, for example, by using time difference of arrival (TDOA) information. To optimize the performance, hybrid data fusion and tracking algorithms can combine both types of sources and further exploit the mobility of the user. Simulation results for different filter types show the ability of this approach to compensate the lack of satellites by additional TDOA measurements from a future 3GPP-LTE communications system. This paper analyzes the performance in a fairly realistic manner by taking into account ray-tracing simulations to generate a coherent environment for GNSS and 3GPP-LTE.
\end{abstract}

\section{Introduction}

Position information of mobile stations (MSs) in a communications system has become a very important feature in recent years. Services and applications based on very accurate location knowledge will play a fundamental role in future wireless systems [1]. Besides the well-known location-based services it is stated by the United States Federal Communications Commission (FCC) that wireless service providers have to deliver the locations of all enhanced 911 (E911) emergency callers with specified accuracy [2]. To meet this requirement, global navigation satellite systems (GNSSs) [3]-like the current Global Positioning System (GPS) and the future European Galileo system-can deliver very good position estimates under optimum conditions. However, especially in critical positioning scenarios with severe multipath propagation and blocking of several satellites by buildings the performance loss can be very high [4]. For instance, in urban canyons the number of visible satellites can be below the required four. In these situations, we propose to include timing measurements from a communications system to compensate the lack of satellites.
As a supplement to GNSS or as stand-alone solution, already available communications systems can be included in the MS localization process. Generally, measurements in terms of time of arrival (TOA), time difference of arrival (TDOA), angle of arrival, or received signal strength, provided by the base stations (BSs) or the MS, can be used [1]. Of very high interest is TDOA positioning as it is already included in several systems (e.g., GSM, UMTS, CDMA2000) $[5,6]$, in the standardization process for 3GPP-LTE (cf. [7-9]), and also under discussion for positioning in future systems (cf. $[10,11])$.

The hybrid data fusion (HDF) of measurements from GNSS and communications systems is important for seamless positioning and navigation in critical environments [12]. The additional utilization of tracking algorithms will further improve the performance for mobile users [13]. In this paper, we investigate a joint HDF and tracking based on different filter types, where we focus on Positioning Kalman filter (PKF), extended Kalman filter (EKF) [14], and particle filter (PF) [15], that combine GNSS measurements with TDOA measurements from a future 3GPP-LTE communications system. Contrary to state-of-the-art work (e.g., [13]), this 
paper analyzes the performance in a fairly realistic manner, especially by taking into account ray-tracing simulations and mobility models for the pedestrian use-case. Since combined channel models for GNSS and communications systems are not yet available, this procedure allows to generate a coherent and correlated environment for both considered systems.

Section 2 starts with a description of the system models for GNSS and the communications system, including also an overview of positioning methods in current and future systems. Section 3 discusses the HDF and tracking algorithms that are employed for this investigation. Finally, simulation results in Section 4 show the performance for the developed approaches in coherent environments for pedestrian positioning in urban canyons.

\section{System Model}

2.1. Global Navigation Satellite Systems. GPS and the future European Galileo system will be considered in our investigations [3]. The distances between the $N_{\mathrm{Sat}}=N_{\mathrm{GPS}}+N_{\text {Galileo }}$ visible satellites located at the positions $x_{\mu}=\left[x_{\mu}, y_{\mu}, z_{\mu}\right]^{\mathrm{T}}, \mu \in$ $\left\{1,2, \ldots, N_{\text {Sat }}\right\}$, which are assumed to be under line-of-sight (LOS) conditions, and the MS at position $x=[x, y, z]^{\mathrm{T}}$ are given by

$$
r_{\mu}(x)=\sqrt{\left(x_{\mu}-x\right)^{2}+\left(y_{\mu}-\mathrm{y}\right)^{2}+\left(z_{\mu}-\mathrm{z}\right)^{2}} .
$$

The resulting pseudoranges between the satellites and MS can then be modeled as

$$
\hat{r}_{\mu}=r_{\mu}(x)+b_{\text {clock }}+b_{\text {multipath }, \mu}+n_{\mu} \text {, }
$$

where $b_{\text {clock }}$ is the clock bias coming from the time-offset of the receiver with respect to the satellite clocks, and $n_{\mu}$ is the elevation depending residual error chosen according to user-equivalent range error (UERE) models for singleband GNSS receivers and effects like tropospheric errors and receiver noise [3]. The multipath error is included in terms of $b_{\text {multipath, } \mu}$. It will be calculated for each channel impulse response (CIR), assuming noncoherent integration and a state-of-the-art narrow early-minus-late correlator with correlator spacing of 0.1 chips, where one chip corresponds to around $300 \mathrm{~m}$ for GPS.

A LOS visibility analysis for GPS and GPS+Galileo created by ray-tracing simulations is shown in Figure 1. It shows the number of LOS-visible satellites in an urban scenario located in Munich, Germany, where the average building height is around $26 \mathrm{~m}$. We see that especially inside the urban canyons there occur several situations, where only less than four satellites are visible. Hence, only limited performance of GNSSs can be expected. Additionally, the impact of multipath propagation to the overall error is comparably high in these situations. Therefore, supplementation from communications systems is of high interest and importance in these GNSS-critical environments.

2.2. Communications Systems. Besides GNSS, also communications systems have the ability to provide positioning information about one or several MSs. Especially in environments with limited GNSS-performance, for example, urban canyons or indoor, communications systems usually have a good coverage. Different principles for obtaining positioning information can be used by communications systems. For instance, measurements in terms of TOA, TDOA, angle of arrival, or received signal strength can be exploited [1]. However, most of the communications systems-already deployed, standardized, or under research-were not foreseen for positioning applications in advance. Hence, the positioning capabilities are usually restricted.

The best performance can be obtained with timing measurements (e.g., [6]), where the key implementation is based on TDOA. They can easily be extracted from system-inherent measurements. The basic idea is to extract the arriving times of signals from several BSs at the MS. To do so, the MS listens to appropriate synchronization channels depending on the system. Then, the TDOAs can be obtained from that. Nevertheless, many challenges have to be overcome in real systems. For instance, for positioning it is assumed that the BSs are fully time and frequency synchronized which is not foreseen in all systems or only an optional feature. However, so-called location measurement units (LMUs) can be used to compute the misalignment of the BSs and provide this information to the network or MS. Furthermore, mobile radio systems are designed in a way that only one strong serving BS should be heard due to spectral efficiency reasons. From a positioning point of view, we need at least three BSs with sufficient signal-tointerference-and-noise ratios (SINRs) for two-dimensional location estimation. This is usually only given at the cell edge. Close to a BS the interference of the serving BS can be too high for detection of the out-of-cell BSs with a reasonable good quality.

Considering deployed systems, in the GSM EDGE radio access network (GERAN), three location principles are specified (cf. [5]): besides the cell identity procedure a TDOAbased method called enhanced observed time difference (E-OTD) is standardized. However, due to large cells in GSM the positioning accuracy can be in the order of several hundred meters. Another specified method is assisted GPS (A-GPS). In assisted solutions, parts of the navigation data-which traditionally is included in the GNSS satellite signals_can be communicated much faster to the MS by the network. This can help to reduce the time-to-first-fix in the acquisition process and to increase the sensitivity in the tracking stage since longer integration times are possible. In CDMA-based systems (e.g., UMTS, W-CDMA, CDMA2000) similar positioning principles as in GSM have been implemented [6]. However, the technical realization is different due to the CDMA properties of the system. On the one hand long integration times can be realized, on the other hand the well-known near-far problem is hard to overcome. However, also here TDOA-based positioning techniques are used to determine the MS position without GNSS support. Additionally, A-GPS approaches are standardized.

The deployment of CDMA-based mobile radio systems is still on-going; nevertheless, the successors are completing the standardization process, where in this paper we focus on 


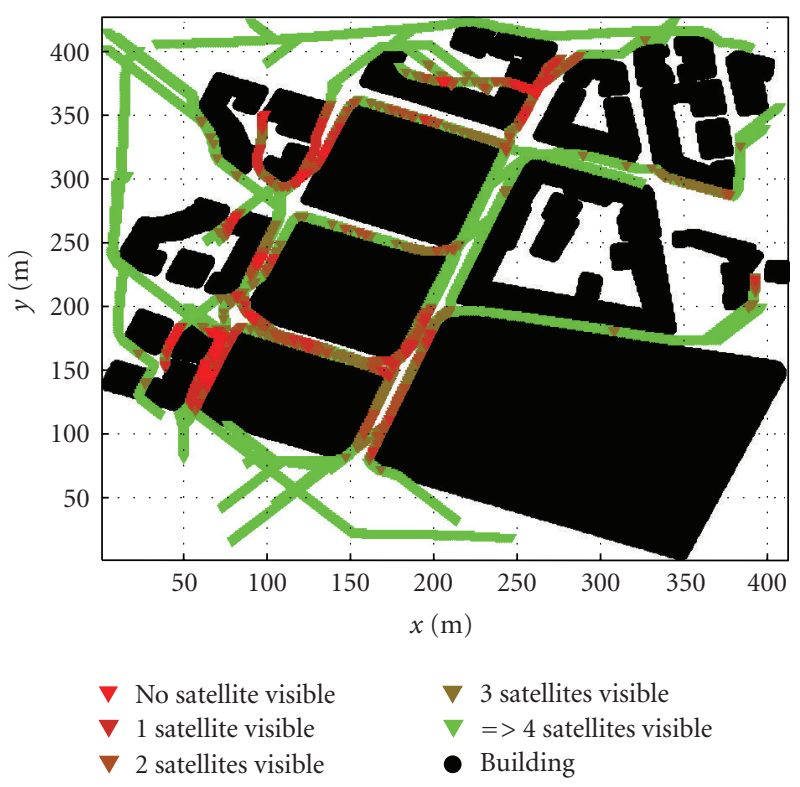

(a) GPS

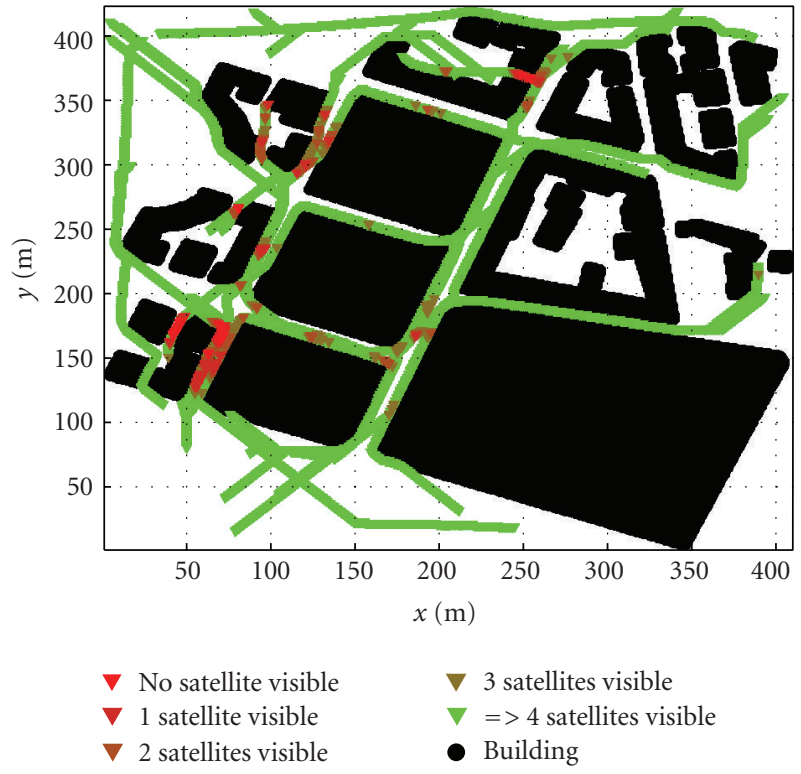

(b) GPS+Galileo

Figure 1: Number of visible GNSS satellites.

3GPP-LTE [7]. 3GPP-LTE will be an orthogonal frequency division multiplexing (OFDM) system with bandwidths up to $20 \mathrm{MHz}$. For timing-based positioning the synchronization and reference channels can be used to determine the TDOAs at the receiver side (e.g., $[8,9])$. Generally, timingbased positioning relies on known sequences in the signals. In 3GPP-LTE the primary and secondary synchronization channels (P-SCH, S-SCH) as well as the downlink reference signal (DL-RS) could be used [16] (cf. Figure 2 for the frame structure of 3GPP-LTE, P-SCH in blue, S-SCH in green, DL-RS in black). For the detection of the different sectors (we assume three sectors per BS) a searching algorithm is applied, which builds replicas of the known synchronization sequences and correlates them with the received signals from all surrounding sectors in time domain to determine the arrival time of the sequences and, thus, to determine finally the TDOAs (e.g., [17]). For our investigations, we simulate a FDD system with $20 \mathrm{MHz}$ bandwidth, directional antennas, and transmit powers of $43 \mathrm{dBm}$ (cf. [17]); however, as specified in the standard, the synchronization sequences only occupy $1 \mathrm{MHz}$ bandwidth.

It can be expected that also the generation of mobile radio systems after 3GPP-LTE will be OFDM based, where the occupied bandwidth will further increase. However, it is questionable if a target bandwidth of $100 \mathrm{MHz}$ (as proposed in [10]) can be realized in spectrum as a whole or only by sophisticated overlay systems. The further integration of local communications systems for hot spot situations will also be challenging from a positioning point of view. On the one hand, the coverage can be really high since an overlap of these hot spots is naturally given. On the other hand, the exact location of these plug-and-play devices is not a priori known which encounters new difficulties in the location determination process. Another concept under discussion is relay enhanced cells, especially in urban environments.

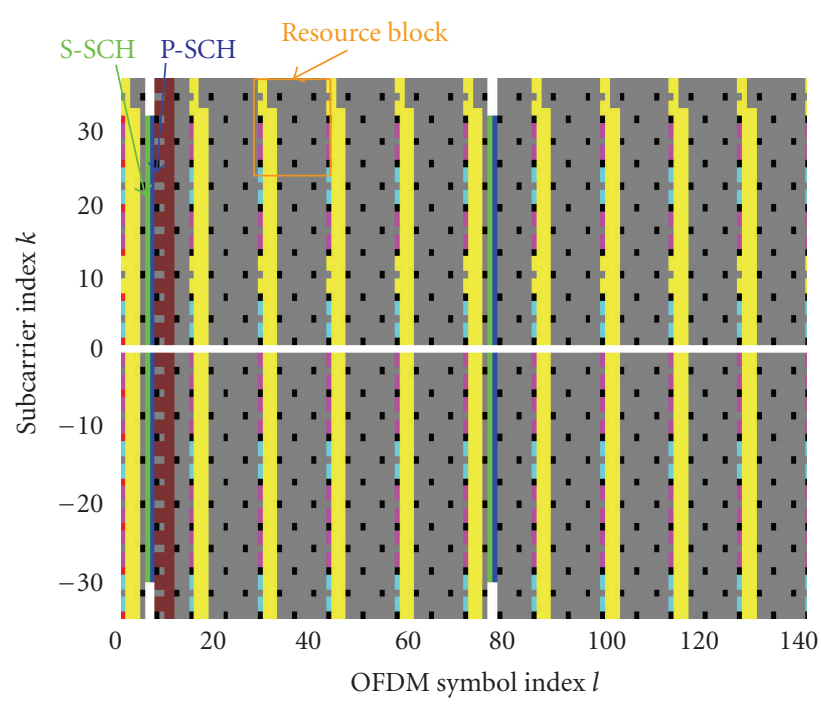

FIGURE 2: 3GPP-LTE frame structure.

As discussed before, for the measurements from the communications part we use TDOAs. In TDOA only timedifferences from several BSs are used; thus, the receiver timeoffset with respect to the synchronized BSs is compensated inherently. Hence, we obtain

$$
d_{v, 1}(x)=r_{\nu}(x)-r_{1}(x), \quad v \in\left\{2,3, \ldots, N_{\mathrm{BS}}\right\},
$$

where we chose BS 1 as reference BS which gives $N_{\mathrm{BS}}-1$ linear independent TDOAs. The TDOA measurement model is determined as

$$
\widehat{d}_{v, 1}=d_{v, 1}(x)+n_{v, 1} .
$$

Each error contribution $n_{v, 1}=n_{v}-n_{1}$ depends on two measurements which results in strong correlations, especially 


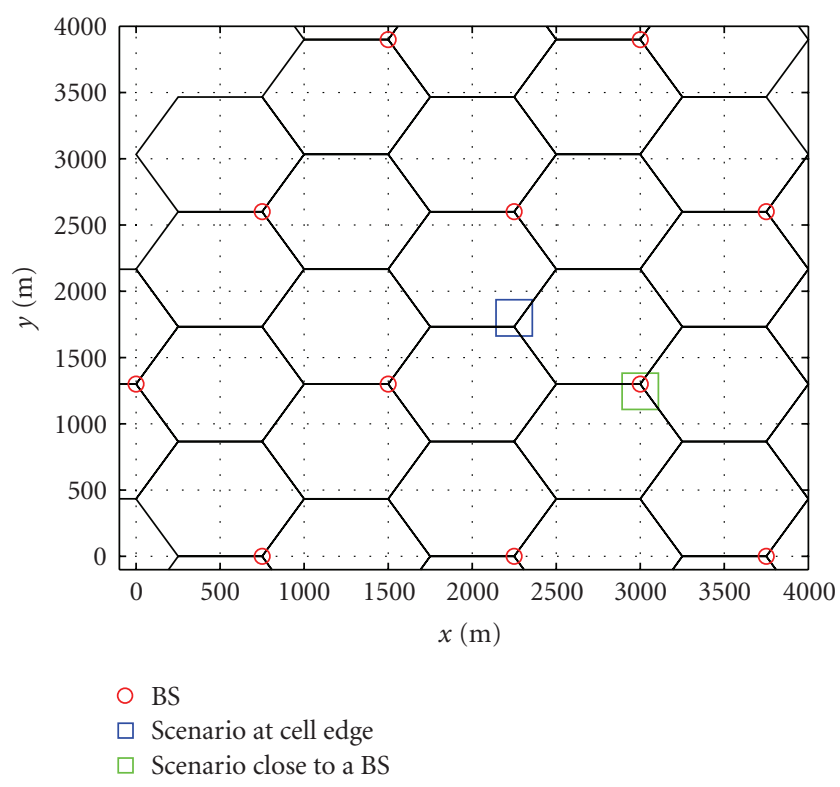

FIgURE 3: Cell layout and simulated scenarios.

with respect to the reference BS. The timing information is extracted from the synchronization algorithms between the MS and several BSs at the same time, where-similar as for GNSS-also here coherent channel conditions are generated (cf. Section 2.3). Note that both multipath and NLOS propagation are considered in the TDOA measurements. However, as multipath is not exploited or mitigated, the multipath effects are not explicitly denoted in this equation; that is, all effects are included in the noise. As pointed out before, we have used a 3GPP-LTE system to provide the TDOAs (e.g., [17]). The cellular network structure is shown in Figure 3, where the urban canyon scenario (cf. Figure 1) is located at two different positions: at the cell edge or close to a BS.

2.3. Channel and Mobility Models. To assess the performance of HDF and tracking algorithms, realistic channel models for GNSSs and communications systems are required, where strong system-, spatial-, and time-correlations occur. No current models meet these requirements in an adequate manner so far. Thus, we created realistic models by using raytracing simulations in an urban canyon scenario to generate CIRs for satellites and BSs at several points. This allows a coherent simulation for the HDF and tracking algorithms. Further, we have adapted and implemented a mobility model that is based on gas diffusion [18]. This model generates realistic path tracks of the MS especially for slow moving pedestrian users. Some of these tracks were already shown in Figure 1. Typical CIRs for such a track assuming a GPS satellite and a BS are depicted in Figure 4. We observe, for example, the high number of reflections and also the clear LOS situations at time-steps $80-170$ and 320-350 for the GPS satellites. Note that for each simulated track the CIRs from all satellites and all BSs are calculated to obtain a coherent and correlated environment.

\section{Hybrid Data Fusion and Tracking Algorithms}

The MS positions are usually correlated over time. For instance-considering a pedestrian-certain information about the position can be derived using the history of past estimates and suitable movement or mobility models. For instance, a pedestrian cannot jump from one position to another in limited time. This behavior can be used as sideinformation for position tracking algorithms.

We assume a hidden Markov model with unknown states $s_{k} \in \mathbb{R}^{N_{s}}$ that have to be estimated in each time-step $k \in \mathbb{N}$. The estimation process takes into account the measurements $y_{k} \in \mathbb{R}^{N_{y}}$ in each time-step $k$ in addition to the model parameters. The measurements $y_{k}$ depend only on the state vector $s_{k}$ at the current time-step. This dependence is defined by the so-called measurement model:

$$
y_{k}=g_{k}\left(s_{k}, n_{k}\right) .
$$

The function $g_{k}$ is a possibly nonlinear function of the state $s_{k}$ and the measurement noise $n_{k} \in \mathbb{R}^{N_{n}}$. The properties of the measurement noise $n_{k}$ define the measurement uncertainties. Another equivalent representation of the measurement model is based on the conditioned probability density function (PDF) of the measurements given the states, that is, $p\left(y_{k} \mid s_{k}\right)$.

The state model defines a relation between the previous state $s_{k-1}$ and the current state $s_{k}$. It is given as

$$
s_{k}=f_{k-1}\left(s_{k-1}, v_{k-1}\right),
$$

where the function $f_{k-1}$ is a possibly nonlinear function of the state $s_{k-1}$ and the state process noise $v_{k-1} \in \mathbb{R}^{N_{v}}$. The properties of the state process noise $v_{k-1}$ define how random the state changes can be. The equivalent representation of the state model is based on the conditioned PDF $p\left(s_{k}\right.$ | $\left.s_{k-1}\right)$. In the positioning context the state vector can include information about the MS position or its velocity. The corresponding state model includes information about the mobility or movement of the MS. Therefore, it is often denoted as mobility model.

Following the Bayesian approach (e.g., $[14,19]$ ), it is required that the PDF of the current state is estimated by considering all previous and the current measurements, that is, the $\operatorname{PDF} p\left(s_{k} \mid y_{1}, y_{2}, \ldots, y_{k}\right)$ has to be constructed. This is done recursively by assuming that the prior distribution of the state $s_{0}$ is known.

In the first step of Bayesian estimation, the state model is used to obtain the prior PDF of the state at time-step $k$ by

$$
\begin{aligned}
& p\left(s_{k} \mid y_{1}, y_{2}, \ldots, y_{k-1}\right)= \\
& \int p\left(s_{k \mid} s_{k-1}\right) p\left(s_{k-1} \mid y_{1}, y_{2}, \ldots, y_{k-1}\right) d s_{k-1} .
\end{aligned}
$$

The PDF $p\left(s_{k} s_{k-1}\right)$ is defined by the state equation and the known statistics of the state noise $v_{k-1}$. This step is denoted as prediction step since the new state is estimated as a prediction of the old state.

For the second step, it is required that at time-step $k$ the measurements $y_{k}$ become available. They can be used 


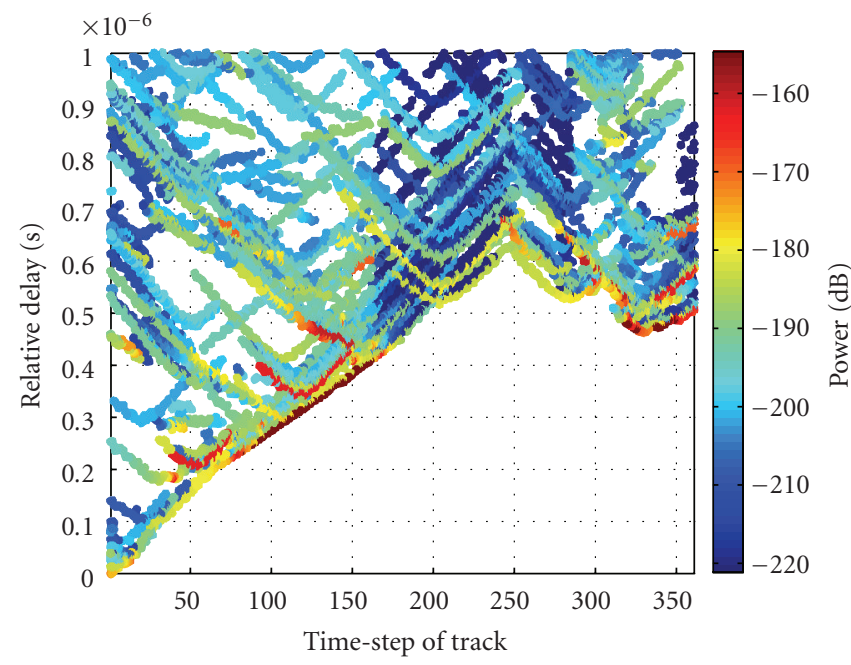

(a) GPS satellite

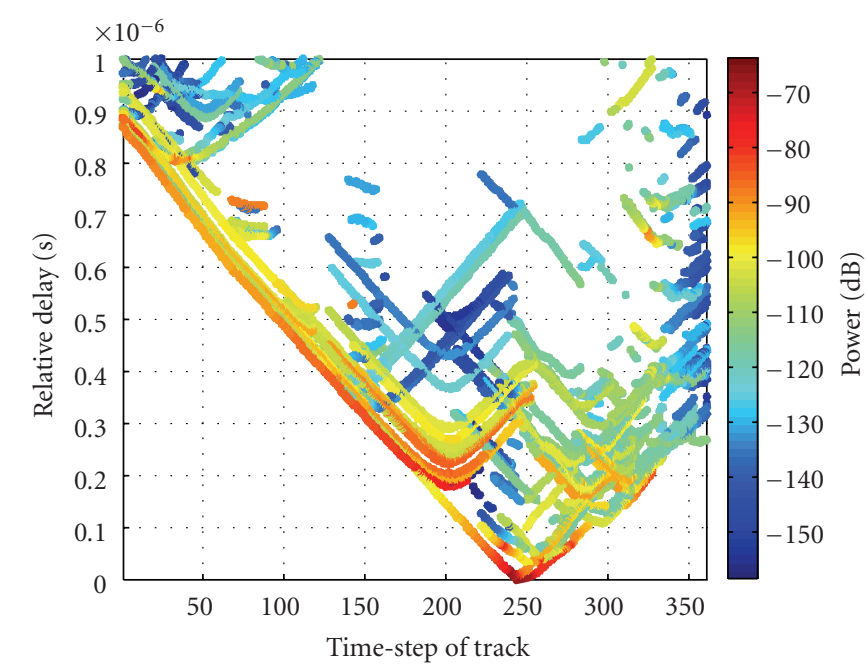

(b) BS

FIGURE 4: CIRs for one track realization.

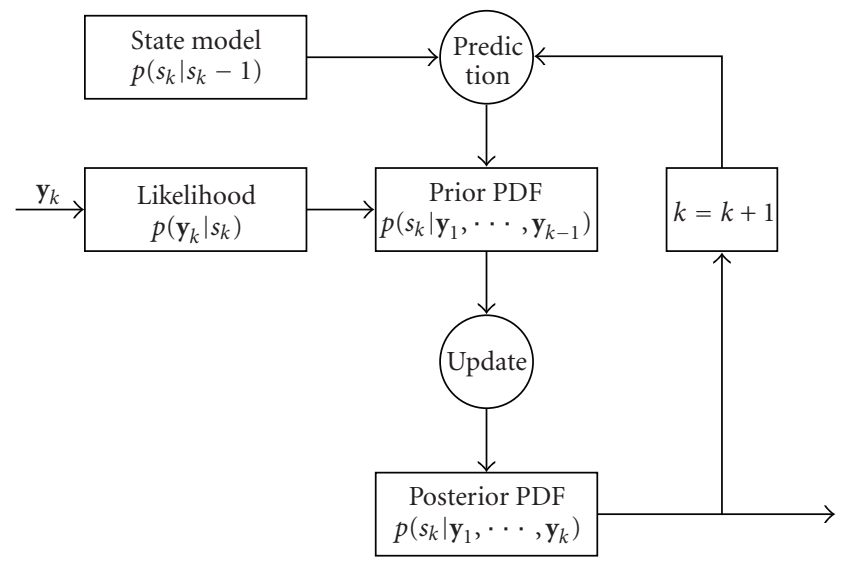

Figure 5: Recursive Bayesian estimation.

to update the prior PDF by the Bayes' rule resulting in a normalized product of the likelihood $p\left(y_{k} \mid s_{k}\right)$ and the prior $\mathrm{PDF}$, that is,

$$
p\left(s_{k} \mid y_{1}, y_{2}, \ldots, y_{k}\right)=\frac{p\left(y_{k} \mid s_{k}\right) p\left(s_{k} \mid y_{1}, y_{2}, \ldots, y_{k-1}\right)}{p\left(y_{k} \mid y_{1}, y_{2}, \ldots, y_{k-1}\right)}
$$

with normalization constant $p\left(y_{k} \mid y_{1}, y_{2}, \ldots, y_{k-1}\right)$. Therefore, the posterior PDF can be calculated by using the measurement model and the known statistics of the measurement noise $n_{k}$. Since the measurements of timestep $k$ are used to modify the prior PDF for obtaining the posterior PDF, this step is called update step. The complete principle of the recursive Bayesian estimator is shown in Figure 5 (cf. [20]).
Finally, the solution that maximizes the posterior PDF is nothing else than the maximum-a-posteriori (MAP) estimator:

$$
\hat{s}_{\mathrm{MAP}, k}=\underset{s_{k}}{\operatorname{argmax}} p\left(s_{k} \mid y_{1}, y_{2}, \ldots, y_{k}\right) .
$$

Contrary to that, the minimum mean square error (MMSE) estimator calculates the expectation over the PDF, that is,

$$
\widehat{s}_{\text {MMSE, }, k}=\int s_{k} p\left(s_{k} \mid y_{1}, y_{2}, \ldots, y_{k}\right) d s_{k},
$$

where for Gaussian noise distributions both estimators yield the same result.

Usually there are no closed-form solutions for the general integrals in Bayes estimation [15]. One option to handle these integrals is certain assumptions for the models or approximations: the classical Kalman filter (KF) approach (Section 3.1) presumes linear models and Gaussian noise distributions. The EKF approximates non-linear models in a linearization step which will be shown in Section 3.2. Another option to handle the integrals is numerical integration. An approximate solution by Monte-Carlo methods is the PF being described in Section 3.3.

3.1. Kalman Filter. The KF (cf. [14]) is one of the most widely used implementation of Bayesian filters. One of the main advantages of KFs is the computational efficiency in the implementation using only matrix and vector operations on the mean and covariances of Gaussian processes.

To perform optimum, it must hold for the system model in (6) and the measurement model in (5) that the system process noise $v_{k} \sim \mathcal{N}\left(0_{N_{s}}, Q_{k}\right)$ and measurement noise $n_{k} \sim \mathcal{N}\left(0_{N_{y}}, C_{k}\right)$ are drawn from zero-mean Gaussian distributions with known covariances. For GNSS the covariance matrix of the measurement noise is based on the error model for each satellite (which is, e.g., elevation dependent); 
for the 3GPP-LTE TDOA measurements it is based on the SINRs. Note that for a general derivation it is not necessarily required that the noise is zero-mean (e.g., [14]) which is assumed here for simplicity. Furthermore, $f_{k-1}$ and $g_{k}$ have to be known linear functions.

Then, we can rewrite (6) and (5) as

$$
\begin{gathered}
s_{k}=A_{k-1} s_{k-1}+v_{k-1}, \\
y_{k}=H_{k} s_{k}+n_{k} .
\end{gathered}
$$

The matrix $A \in \mathbb{R}^{N_{s} \times N_{s}}$ is the state matrix and includes the linear dependencies between the states of time-steps $k$ and $k-1$. The measurement matrix $H \in \mathbb{R}^{N_{y} \times N_{s}}$ reflects the linear relation between the measurements and the state at time-step $k$. In general, all matrices can be time-variant. In the context of positioning applications this could reflect, for example, changing mobility models over time. The optimum filter equations can then be written as follows.

In a first step (prediction) the state of the current timestep is calculated taking into account the state of the previous time-step and the knowledge of the state matrix given by $A_{k}$. Then, the estimate of the state after prediction is

$$
\widehat{s}_{k \mid k-1}=A_{k-1} \hat{s}_{k-1 \mid k-1}
$$

with the estimate of the previous time-step $\widehat{s}_{k-1 k-1}$. Additionally, the corresponding MMSE or covariance matrix after that prediction step can be calculated as

$$
M_{k \mid k-1}=A_{k-1} M_{k-1 \mid k-1} A_{k-1}^{T}+Q_{k}
$$

where $M_{k-1 \mid k-1}$ is the MMSE matrix of the previous timestep. From the Bayesian PDF point of view, the prior PDF can be represented as a Gaussian distribution according to

$$
p\left(s_{k} \mid y_{1}, y_{2}, \ldots, y_{k-1}\right) \sim \mathcal{N}\left(\hat{s}_{k \mid k-1}, M_{k \mid k-1}\right) .
$$

The Kalman gain matrix includes a weighting between the predicted estimate (already calculated) and the current measurements. It is given by

$$
K_{k}=M_{k \mid k-1} H_{k}^{\mathrm{T}}\left(C_{k}+H_{k} M_{k \mid k-1} H_{k}^{\mathrm{T}}\right)^{-1} .
$$

Finally, the correction step combines the predicted estimates with the current measurements weighted with the Kalman gain matrix. This results in the final estimate of the state vector:

$$
\hat{s}_{k \mid k}=\hat{s}_{k \mid k-1}+K_{k}\left(y_{k}-H_{k} \hat{s}_{k \mid k-1}\right) .
$$

The corresponding MMSE or covariance matrix after the correction step is obtained as

$$
M_{k \mid k}=\left(\mathbf{I}_{N_{s}}-K_{k} H_{k}\right) M_{k \mid k-1} .
$$

The resulting posterior PDF can then be written as Gaussian distribution according to

$$
p\left(s_{k} \mid y_{1}, y_{2}, \ldots, y_{k}\right) \sim \mathcal{N}\left(\hat{s}_{k \mid k}, M_{k \mid k}\right)
$$

The KF is initialized with $s_{0 \mid 0}$ and $M_{0 \mid 0}$ determined by the prior distribution of the initial state.

In the context of position tracking applications, we assume that the state vector consists of position and velocity, that is,

$$
s_{k}=\left[x_{k}, y_{k}, z_{k}, v_{x, k}, v_{y, k}, v_{z, k}\right]^{\mathrm{T}} \text {. }
$$

For the mobility model, we choose a very simple model corresponding to the principle of random walk. For that, the resulting time-invariant system matrix is given by

$$
A=\left[\begin{array}{llllll}
1 & 0 & 0 & T & 0 & 0 \\
0 & 1 & 0 & 0 & T & 0 \\
0 & 0 & 1 & 0 & 0 & T \\
0 & 0 & 0 & 1 & 0 & 0 \\
0 & 0 & 0 & 0 & 1 & 0 \\
0 & 0 & 0 & 0 & 0 & 1
\end{array}\right]
$$

The sampling time $T$ depends on the application. For instance, for pedestrian positioning sampling times of around $1 \mathrm{~s}$ are usually sufficient. The covariance matrix of the process noise is a diagonal matrix including the variance of the mobility (process drift) in all directions for position and velocity. This model implies that the change of the MS position is controlled by process noise of a certain variance.

For the measurement model, we assume that in every time-step a static position estimate is available. The static solution (cf. [12]) results in a weighted non-linear least squares estimation problem. As an analytic solution is not possible, an iterative procedure based on the Gauss-Newton algorithm is applied. Hence, for the Kalman equations the measurements are given in terms of position measurements and have a linear dependency with the state vector which is reflected in the time-invariant measurement matrix:

$$
H=\left[\begin{array}{llllll}
1 & 0 & 0 & 0 & 0 & 0 \\
0 & 1 & 0 & 0 & 0 & 0 \\
0 & 0 & 1 & 0 & 0 & 0
\end{array}\right] .
$$

We do not consider any velocity estimates which are available from the position estimation entity. Thus, the velocity is handled as hidden state and estimated implicitly in the filter equations. The resulting filter in the positioning context is denoted as positioning KF (PKF).

3.2. Extended Kalman Filter. The performance of the PKF is optimum if the conditions on Gaussianity and linearity are fulfilled completely. Further, the KF requires that the underlying entity which provides the static solutions performs optimum; that is, in each time-step enough sources (here, at least three) have to be available and the static position estimation errors have further to fulfill the Gaussian assumption. Especially in critical positioning situations like urban canyons or indoor, it may happen quite often that only less than the required number of sources are available for a certain time. Then, the PKF would totally fail since the static solution cannot provide any estimates. 
The EKF (e.g., $[14,21])$ is a much more flexible tool being able to handle directly non-linear models. We assume that the state model is given by

$$
s_{k}=a_{k-1}\left(s_{k-1}\right)+v_{k-1}
$$

and the measurement model can be written as

$$
y_{k}=h_{k}\left(s_{k}\right)+n_{k} .
$$

Basic idea of the EKF is a linearization of $a_{k-1}\left(s_{k-1}\right)$ about the estimate of $s_{k-1}$. We obtain

$$
a_{k-1}\left(s_{k-1}\right) \approx a_{k-1}\left(\hat{s}_{k-1 \mid k-1}\right)+A_{k-1}\left(s_{k-1}-\hat{s}_{k-1 \mid k-1}\right)
$$

with the Jacobian matrix:

$$
A_{k-1}=\left.\frac{\partial a_{k-1}\left(s_{k-1}\right)}{\partial s_{k-1}}\right|_{s_{k-1}=\hat{s}_{k-1 \mid k-1}} .
$$

Equivalently, we linearize $h_{k}\left(s_{k}\right)$ about the estimate of $s_{k}$, that is,

$$
h_{k}\left(s_{k}\right) \approx h_{k}\left(\hat{s}_{k \mid k-1}\right)+H_{k}\left(s_{k}-\hat{s}_{k \mid k-1}\right)
$$

with the Jacobian matrix:

$$
H_{k}=\left.\frac{\partial h_{k}\left(s_{k}\right)}{\partial s_{k}}\right|_{s_{k}=\hat{s}_{k \mid k-1}} .
$$

Obviously, the Jacobians have to be recalculated in every time-step since they depend on the estimates of the previous time-steps. However, the resulting structure of the EKF as pointed out in the following is very similar to the PKF solution.

It starts with the prediction, where knowledge of the MS movement model is applied to obtain

$$
\widehat{s}_{k \mid k-1}=a_{k}\left(\widehat{s}_{k \mid k-1}\right),
$$

with the estimate of the previous time-step $\hat{s}_{k-1 \mid k-1}$. Similarly, the corresponding MMSE or covariance matrix after that prediction step is

$$
M_{k \mid k-1}=A_{k-1} M_{k-1 \mid k-1} A_{k-1}^{T}+Q_{k}
$$

Due to the linearization step the resulting estimated prior PDF in the Bayesian sense is a Gaussian approximation of the true prior PDF. Hence, the estimated prior PDF is given as

$$
p\left(s_{k} \mid y_{1}, y_{2}, \ldots, y_{k-1}\right) \sim \mathcal{N}\left(\hat{s}_{k \mid k-1}, M_{k \mid k-1}\right) .
$$

The Kalman gain matrix can be obtained by

$$
K_{k}=M_{k \mid k-1} H_{k}^{T}\left(C_{k}+H_{k} M_{k \mid k-1} H_{k}^{T}\right)^{-1},
$$

Finally, the correction step combines the predicted estimates with the current measurements weighted with the Kalman gain matrix. This results in the final estimate of the state vector being calculated as

$$
\widehat{s}_{k \mid k}=\widehat{s}_{k \mid k-1}+K_{k}\left(\mathrm{y}_{k}-h\left(\hat{s}_{k \mid k-1}\right)\right)
$$

The corresponding MMSE or covariance matrix after correction is obtained as

$$
M_{k \mid k}=\left(\mathbf{I}_{N_{s}}-K_{k} H_{k}\right) M_{k \mid k-1} .
$$

Also the resulting posterior PDF is a Gaussian distribution of the true posterior PDF. It is given as

$$
p\left(s_{k} \mid y_{1}, y_{2}, \ldots, y_{k}\right) \sim \mathcal{N}\left(\widehat{s}_{k \mid k}, M_{k \mid k}\right) .
$$

Compared to the PKF, the EKF has no optimality properties, where its accuracy depends on the accuracy of the linearization. Nevertheless, the EKF turns out to be a flexible and robust approach widely used for position tracking applications.

Considering position tracking (as shown for the PKF in Section 3.1), the state vector $s_{k}$ is only extended by 
the unknown clock offset between GNSS satellites and

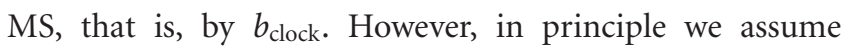
the same state model, that is, a linearization of the state equation is not necessary. Therefore, the prediction step is similar for EKF and PKF. As measurements we process directly the pseudorange measurements from GNSS and the TDOA measurements from the communications systemin contradiction to the PKF, where the measurements were processed beforehand by a static position estimation. Since the measurements are non-linear with respect to position, for the update step a linearization of the measurement model is necessary. For that, the Jacobian matrix including the derivatives of the measurement model with respect to the state vector has to be available. For the considered set-up, $H_{k}$ is shown in (31) with the corresponding indices for satellites (Sat) and BSs (BS).

3.3. Particle Filter. Another important class of Bayesian filters is based on approximation of integrals by numerical integration. These methods are commonly denoted as PFs ([15, 19, 22]) and became quite popular for position tracking applications (e.g., [23]). PFs are based on a sequential Monte-Carlo methodology (cf. [24]) and calculate recursively the relevant PDFs by importance sampling and approximation of PDFs with discrete random measures. Compared to KFs the PFs have usually a much higher complexity depending on the number of particles that have to be generated to model the required PDFs. In addition, they can suffer from phenomena like sample degeneracy or sample impoverishment causing unstable behavior.

In PFs, the posterior PDF is represented as the weighted sum:

$$
p\left(s_{k} \mid y_{1}, y_{2}, \ldots, y_{k}\right)=\sum_{i=1}^{N_{p}} w_{k}^{i} \delta\left(s_{k}-s_{k}^{i}\right),
$$

where each particle consists of a state $s_{k}^{i}$ and a weight $w_{k}^{i}$, and $\delta(\cdot)$ is the Dirac delta measure. The particles are drawn according to the principle of importance sampling from a proposal density $q\left(s_{k} \mid s_{k}^{i}, y_{k}\right)$. The corresponding weights can then be calculated by

$$
w_{k}^{i} \sim w_{k}^{i-1} \frac{p\left(y_{k} \mid s_{k}^{i}\right) p\left(s_{k}^{i} \mid s_{k}^{i-1}\right)}{q\left(s_{k} \mid s_{k}^{i}, y_{k}\right)} .
$$

The generic PF applies the optimum proposal density which in practice is difficult to use. Therefore, often the so-called sampling importance resampling PF (SIR-PF) is implemented (cf. $[15,19,25])$. It only requires that the state and measurement functions $f_{k}$ and $g_{k}$ are known and that sampling of realizations from the state noise distribution of $v_{k-1}$ as well as the prior distribution is possible. In addition, the likelihood function $p\left(s_{k \mid} y_{k}\right)$ has to be available for pointwise evaluation.

In the first step of SIR-PF, for each particle $i=$ $1,2, \ldots, N_{p}$, a sample from the proposal density has to be drawn, that is,

$$
s_{k}^{i} \sim p\left(s_{k} \mid s_{k-1}^{i}\right)
$$

This can be realized by generating a state noise sample $v_{k-1}^{i}$ with the corresponding PDF $p_{v}\left(v_{k-1}\right)$ and setting

$$
s_{k}^{i}=f_{k-1}\left(s_{k-1}^{i}, v_{k-1}^{i}\right) \text {. }
$$

In a second step, for each particle the weights have to be calculated. With the chosen proposal density, this step reduces to

$$
w_{k}^{i}=p\left(y_{k} \mid s_{k}^{i}\right)
$$

Finally, all weights have to be normalized by

$$
w_{k}^{i}=\frac{w_{k}^{i}}{W}
$$

using

$$
W=\sum_{i=1}^{N_{p}} w_{k}^{i}
$$

A crucial problem of the PF is the degeneracy phenomenon $([15,19])$. It points out that after a few iterations, all but one particle will have weights very close to zero. Simply using a very large number of particles is often too inefficient from a computational complexity point of view. A much better method is the application of resampling where degeneracy can be reduced remarkably. The idea is an elimination of particles with low weights to concentrate on particles having large weights. In this manner, a new set of states $\tilde{s}_{k}^{i}, i=1,2, \ldots, N_{p}$ is created by resampling $N_{p}$ times from an approximate discrete representation of

$$
p\left(s_{k} \mid y_{1}, y_{2}, \ldots, y_{k}\right) \approx \sum_{i=1}^{N_{p}} w_{k}^{i} \delta\left(s_{k}-s_{k}^{i}\right) .
$$

Given

$$
P\left(\widetilde{s}_{k}^{j}=s_{k}^{i}\right)=w_{k}^{j}
$$

the resulting sample is an i.i.d. sample from the discrete density. Even though the degeneracy can be reduced by resampling, another effect denoted as sample impoverishment is introduced in practical implementations. Besides the problem of limited parallelization due to the fact that the particles have to be combined, particles with large weights are statistically selected much more often than the other particles. So the diversity among the particles is reduced since the resulting sample will contain many repeated points. Especially for systems with small state noise the sample impoverishment can be a serious problem and all particles can be concentrated to a single state after a few iterations.

In addition to the SIR-PF, there exist several other PF approaches in the literature. Briefly mentioned at this point should be the auxiliary sampling importance resampling PF [26] or the regularized PF [27].

Note that in this paper, the PF is not used as an object of research but solely as lower bound for our positioning algorithms. For that we have used the SIR-PF implementation using 10000 particles. 


\section{Simulation Results}

We assume a fixed GNSS constellation during one track and two different BS constellations. The first scenario is close to a $\mathrm{BS}$, and the second scenario is at the cell edge (cf. Figure 3 ). We assume a cellular network with inter-BS distance of $1500 \mathrm{~m}$, three sectors for each BS, and 3GPP-LTE parameters following the on-going standardization process [16].

We start with simulation results for positioning with GNSS. Figure 6 shows the performance of different GNSS systems averaged over several noise realizations and several generated tracks. The cumulative distribution function (CDF) shows the probability that the position estimation error is below a certain value $x$. In particular, all simulations are performed for the 50 tracks shown in Figure 1 of 290 time-steps each. For each track 100 noise realizations were generated. All in all this results in 1450000 simulated estimates for creating the CDFs. As comparison, the curves for an optimum free space situation are shown in this plot as well. We observe very high performance losses in the urban canyon scenario compared to the free space situation, which comes from lack of satellites and high multipath impacts. However, we can also see that the EKF clearly outperforms the static solution for this situation. Furthermore, the large benefit of additional Galileo satellites with respect to GPSonly positioning becomes obvious.

Figure 7 compares the performance in the urban canyon scenario for different tracking filter types using GPS+Galileo. We observe that with the static solution an accuracy of better than $40 \mathrm{~m}$ can be achieved in $90 \%$ of the cases. With the PKF this can be improved to around $27 \mathrm{~m}$. Using an EKF implementation, we can achieve around $9 \mathrm{~m}$ which comes very close to the performance bound of the PF. For this GNSS-only scenario this is not surprising as the requirement on Gaussianity is met and the nonlinearities are not severe.

Next, the quality of the TDOA measurements is analyzed in detail. Figure 8 shows the investigation for the urban canyon scenario at the cell edge using different filter types when processing two TDOA measurements (i.e., three BSs). As expected, the accuracy as well as the availability is worse compared to GNSS positioning. Nevertheless, using the PF a $90 \%$-accuracy of around $40 \mathrm{~m}$ can be achieved. The EKF provides a slightly worse performance in terms of availability; with static solution and PKF no precise positioning is possible. Note that if the scenario is close to a BS (not shown here), the performance becomes even worse. In that situation also EKF and PF cannot provide a reliable position estimates. Reason for this behavior is that close to a BS usually out-of-cell BSs cannot be received with sufficient quality due to the strong interference of the serving BS. In these situations either a change in the standard is necessary (e.g., 3GPP-LTE proposes a positioning reference mode with idle periods and extended pilot grid [28]) or advanced receiver techniques have to be applied (e.g., [17] proposes to use interference cancellation to increase hearability of out-of-cell BSs). From Figures 7 and 8 it can be concluded that the EKF is a sufficiently good estimator for the considered scenario and the-much more complex-PF gives no fundamental

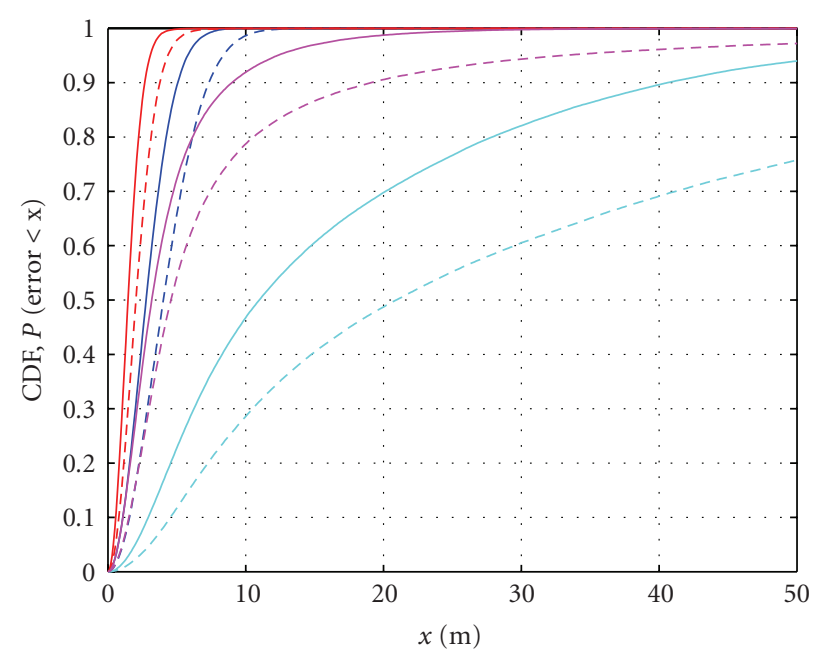

- - - Free space, GPS, static solution

- Free space, GPS + Galileo, static solution

- - - Free space, GPS, EKF

— Free space, GPS + Galileo, EKF

- Urban canyon, GPS, static solution

_ Urban canyon, GPS + Galileo, static solution

- - - Urban canyon, GPS, EKF

_ Urban canyon, GPS + Galileo, EKF

FIGURE 6: GNSS positioning, different scenarios.

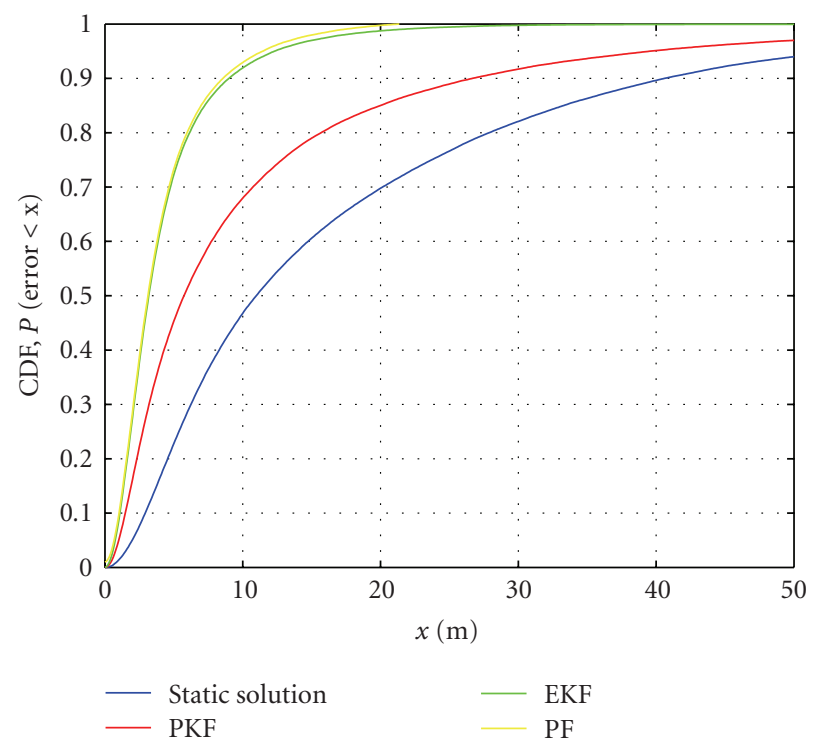

FIGURE 7: GNSS positioning in urban canyon, different tracking filters.

additional performance gains. Hence, in the following we focus on HDF approaches using EKF tracking.

When using stand-alone TDOA positioning it is reasonable to use two TDOAs (i.e., three BSs) for position estimation since this is the minimum number of required measurements. However, when we fuse GNSS with TDOA measurements, simulations have shown that an adaptive algorithm can help to improve the performance. Since the 


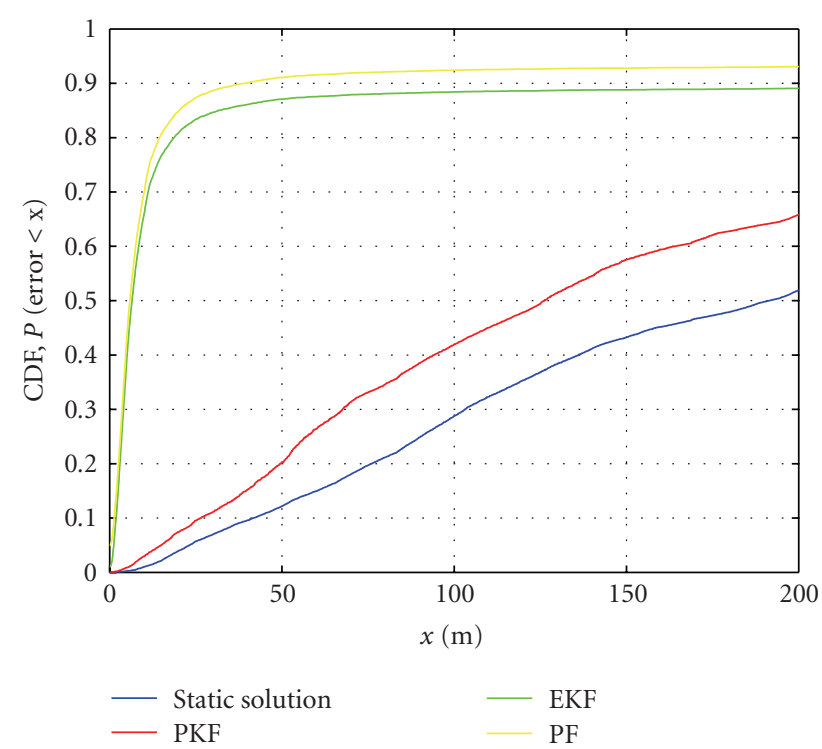

Figure 8: TDOA positioning in urban canyon, cell edge, different tracking filters.

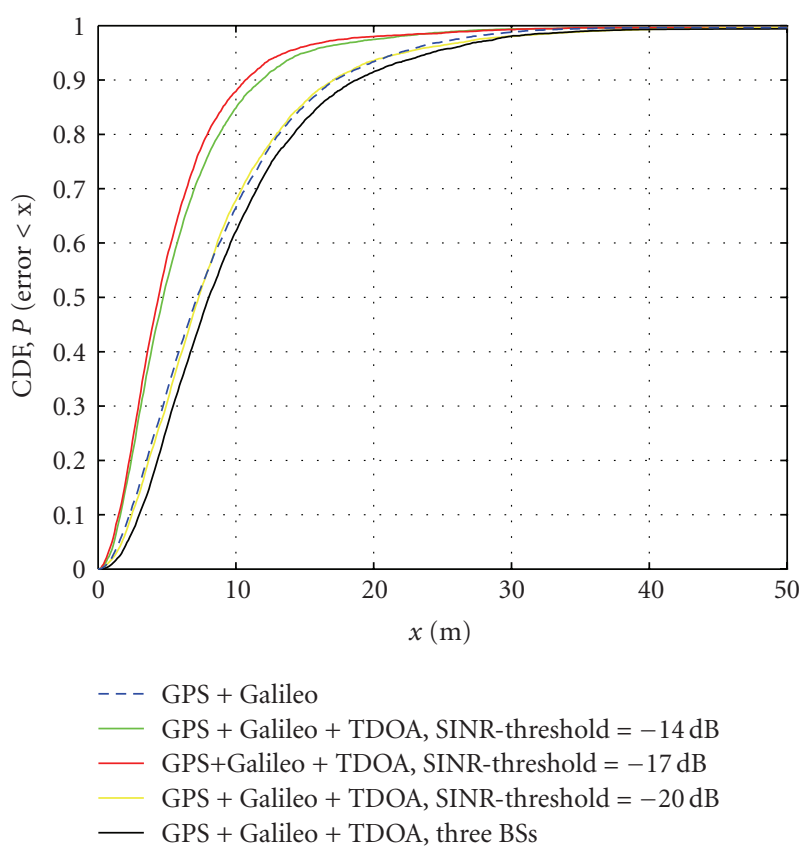

FIGURE 9: HDF in critical situations, cell edge, EKF tracking, different SINR-thresholds.

tracking filters allow a flexible number of measurements in each time-step, we can adapt the number according to the received SINR levels. Therefore, we define an SINRthreshold, where we drop all measurements that are below this threshold. Hence, unreliable TDOA estimates are not used for the HDF process. Note that the weighting is only based on the SINRs; however, also multipath and especially NLOS propagation have a high impact on the TDOA estimates which are not completely reflected by the SINRs. As we do not assume to know the CIRs or do not consider

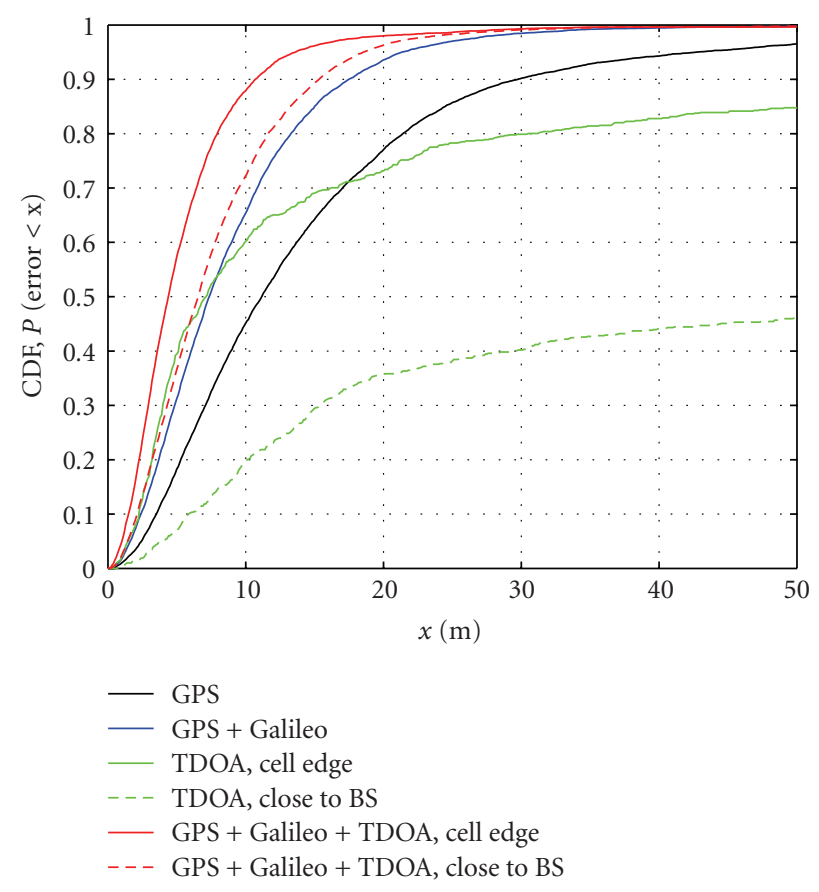

FIGURE 10: Different positioning systems in critical situations, EKF tracking.

any multipath mitigation, the SINR-thresholding results in different performances. The simulation results in Figure 9 show that an SINR-threshold of $-17 \mathrm{~dB}$ is a good choice for the considered scenarios, achieving nearly $10 \mathrm{~m}$ for the $90 \%$-accuracy in this scenario using GPS+Galileo+TDOA compared to around $17 \mathrm{~m}$ for only GPS+Galileo. The adaptive approach which uses the SINR-thresholding also clearly outperforms the classical approach that uses a fixed number of three BSs.

Figure 10 shows the location estimation performance for the GNSS critical situations, where only less than four satellites are available. In these situations, cellular support is particular beneficial. Nevertheless, for standalone cellular positioning we observe the high dependency on the MS position in the scenario. At the cell edge a reasonable performance can be achieved, whereas close to the BS the accuracy is very low. Investigating the classical GNSS-based navigation, we observe the advantage of the additional Galileo satellites compared to GPS-stand-alone positioning. The $90 \%$-accuracy can be reduced from $30 \mathrm{~m}$ to around $18 \mathrm{~m}$ using both systems. When we further include TDOA measurements from the communications system, the performance can be improved to around $15 \mathrm{~m}$ if we are close to the BS and to below $11 \mathrm{~m}$ for the cell edge situation.

In Figure 11, the LOS visibility of all GNSS satellites is plotted over time for one characteristic MS track realization. We see that in several situations the number of LOS-visible and, hence, detectable satellites is below four; that is, we have a critical scenario. Figure 12 shows the corresponding root mean square errors (RMSEs) over time for GPS+Galileo and the TDOA supplements. It can be observed that in critical GNSS situations the RMSE increases as expected. 


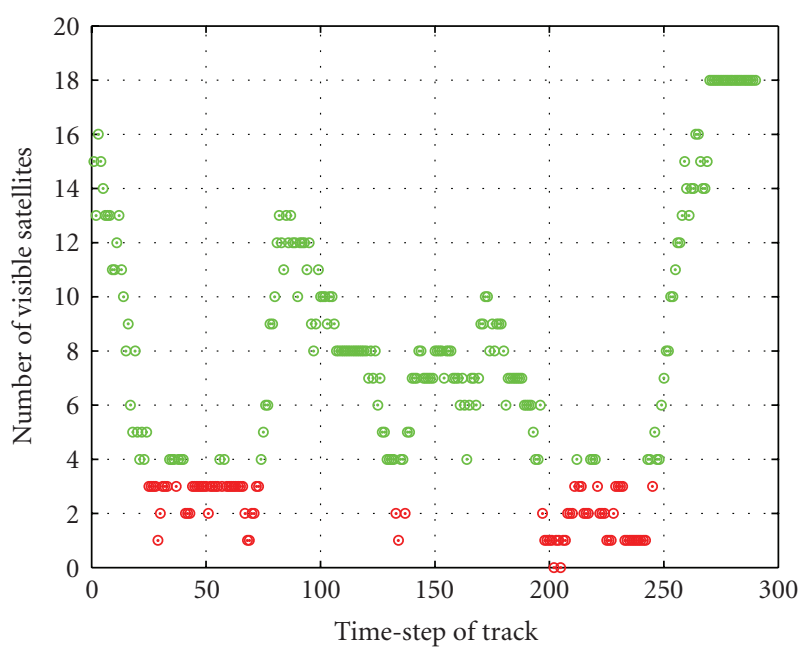

FIGURE 11: Number of visible satellites versus time for one track.

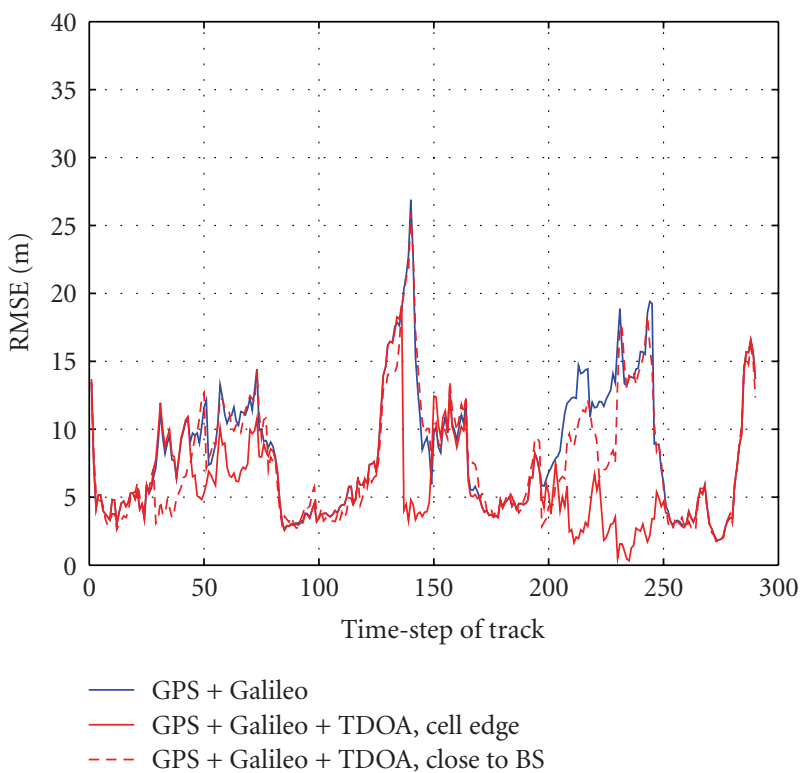

FIGURE 12: RMSE versus time with different positioning systems for one track, EKF tracking.

This comes on the one hand from the limited number of visible satellites. On the other hand, these satellites are affected by severe multipath contributions that cause additional biases in the timing measurements. Furthermore, the geometric constellation of these satellites is usually bad as satellites are only visible above the MS. In this situation the TDOA measurements can compensate the lack of satellites and further improve the geometric conditions. However, if the scenario is close to a BS, the performance gain is restricted and no fundamental improvement to satellite-only positioning can be achieved. For the cell edge situation, the RMSE can be reduced remarkably in the considered critical situations.

\section{Conclusions}

In this paper we have analyzed hybrid positioning methods that combine measurements from GNSS and future 3GPPLTE communications systems. As expected, the performance depends strongly on the location in the network, that is, cell edge or close to a BS, where TDOA measurements are exploited for stand-alone positioning and to support GNSS in critical urban canyon scenarios. The simulations were performed in a fairly realistic manner. The urban environment was reflected by CIRs from the satellites and BSs, which were obtained by ray-tracing. Simulation results in this coherent and correlated scenario have shown that in situations where several satellites are blocked, it is very beneficial to have additional TDOA measurements to compensate the missing satellites. Besides the static solution, different tracking filters were investigated, in particular PKF, EKF, and PF. The simulations showed that the EKF provides the best trade-off between accuracy and complexity for the considered scenarios.

\section{Acknowledgments}

This work has been performed in the projects WHERE (Grant Agreement no. 217033) and GRAMMAR (Grant Agreement no. 227890), which are partly funded by the European Union under the European Community's Seventh Framework Programme (FP7/2007-2013), and the DLRinternal project Galileo Advanced Applications. The material in this paper was partly presented at the International Workshop on Synergies in Communications and Localization (SyCoLo), Dresden, Germany, June 2009.

\section{References}

[1] A. H. Sayed, A. Tarighat, and N. Khajehnouri, "Network-based wireless location,” IEEE Signal Processing Magazine, vol. 22, no. 4, pp. 24-40, 2005.

[2] "FCC 99-245: third report and order," Tech. Rep., Federal Communications Commission (FCC), Rickey Davis, Ala, USA, October 1999, http://www.fcc.gov/911/enhanced/.

[3] P. Misra and P. Enge, Global Positioning System: Signals, Measurements and Performance, Ganga-Jamuna Press, Lincoln, Mass, USA, 2004.

[4] R. Ercek, P. De Doncker, and F. Grenez, "Study of pseudorange error due to non-line-of-sight-multipath in urban canyons," in Proceedings of the 18th International Technical Meeting of the Satellite Division of The Institute of Navigation (ION GNSS '05), pp. 1083-1094, September 2005.

[5] Y. Zhao, "Mobile phone location determination and its impact on intelligent transportation systems," IEEE Transactions on Intelligent Transportation Systems, vol. 1, no. 1, pp. 55-64, 2000.

[6] Y. Zhao, "Standardization of mobile phone positioning for 3G systems," IEEE Communications Magazine, vol. 40, no. 7, pp. 108-116, 2002.

[7] Third Generation Partnership Project (3GPP), 2009, http:// www.3gpp.org.

[8] 3GPP, Qualcomm Europe, "R1-090353: On OTDOA in LTE," 3GPP TSG-RAN WG1 \#55bis, January 2009.

[9] 3GPP, Nortel, "R1-090765: LTE Neighbor Cell Hearability," 3GPP TSGRAN WG1 \#56, February 2009. 
[10] Wireless World Initiative New Radio (WINNER) Project, September 2008, https://www.ist-winner.org.

[11] IST-2003-507581, "WINNER Deliverable D4.8.1: WINNER II Intramode and Intermode Cooperation Schemes Definition," June 2006, https://www.ist-winner.org.

[12] F. Dovis, P. Mulassano, and D. Orgiazzi, "Assessment study of navigation and communication terminal-based hybrid positioning," in Proceedings of the International Technical Meeting of the Satellite Division of The Institute of Navigation (ION/GNSS '02), May 2002.

[13] T. Perälä and R. Piché, "Robust extended Kalman filtering in hybrid positioning applications," in Proceedings of the 4th Workshop on Positioning, Navigation and Communication (WPNC '07), pp. 55-63, March 2007.

[14] S. M. Kay, Fundamentals of Statistical Signal Processing: Estimation Theory, Prentice Hall, New York, NY, USA, 1993.

[15] M. S. Arulampalam, S. Maskell, N. Gordon, and T. Clapp, "A tutorial on particle filters for online nonlinear/non-Gaussian Bayesian tracking," IEEE Transactions on Signal Processing, vol. 50, no. 2, pp. 174-188, 2002.

[16] 3GPP TS 36.211, "Evolved Universal Terrestrial Radio Access (E-UTRA)_Physical Channels and Modulation, V8.8.0," September 2009, http://www.3gpp.org.

[17] C. Mensing, S. Sand, A. Dammann, and W. Utschick, "Interference-aware location estimation in cellular OFDM communications systems," in Proceedings of the IEEE International Conference on Communications (ICC '09), June 2009.

[18] J. Kammann, M. Angermann, and B. Lami, "A new mobility model based on maps," in Proceedings of the IEEE 58th Vehicular Technology Conference (VTC '03), pp. 3045-3049, October 2003.

[19] B. Ristic, S. Arulampalam, and N. Gordon, Beyond the Kalman Filter-Particle Filters for Tracking Applications, Artech House, Norwood, Mass, USA, 2004.

[20] B. Krach, M. Lentmaier, and P. Robertson, "Bayesian detection and tracking for joint positioning and multipath mitigation in GNSS," in Proceedings of the 5th Workshop on Positioning, Navigation and Communication (WPNC '08), pp. 173-180, March 2008.

[21] P. Maybeck, Stochastic Models, Estimation and Control, vol. 1, Academic Press, New York, NY, USA, 1979.

[22] P. M. Djurić, J. H. Kotecha, J. Zhang et al., "Particle filtering," IEEE Signal Processing Magazine, vol. 20, no. 5, pp. 19-38, 2003.

[23] F. Gustafsson, F. Gunnarsson, N. Bergman et al., "Particle filters for positioning, navigation, and tracking," IEEE Transactions on Signal Processing, vol. 50, no. 2, pp. 425-437, 2002.

[24] A. Doucet, J. F. G. de Freitas, and N. J. Gordon, "An introduction to sequential Monte Carlo methods," in Sequential Monte Carlo Methods in Practice, A. Doucet, J. F. G. de Freitas, and N. J. Gordon, Eds., Springer, New York, NY, USA, 2001.

[25] N. J. Gordon, D. J. Salmond, and A. F. M. Smith, "Novel approach to nonlinear/non-Gaussian Bayesian state estimation," Proceedings the IEE, vol. 140, no. 2, pp. 107-113, 1993.

[26] M. K. Pitt and N. Shephard, "Filtering via simulation: auxiliary particle filters," Journal of the American Statistical Association, vol. 94, no. 446, pp. 590-599, 1999.

[27] C. Musso, N. Oudjane, and F. LeGland, "Improving regularized particle filters," in Sequential Monte Carlo Methods in Practice, A. Doucet, J. F. G. de Freitas, and N. J. Gordon, Eds., Springer, New York, NY, USA, 2001.
[28] 3GPP, Ericsson, ST-Ericsson, Qualcomm, "R4-093400: OTDOA positioning studies in RAN4: Updated Proposal on System Simulation Assumptions," 3GPP TSG-RAN WG4 \#52, August 2009. 

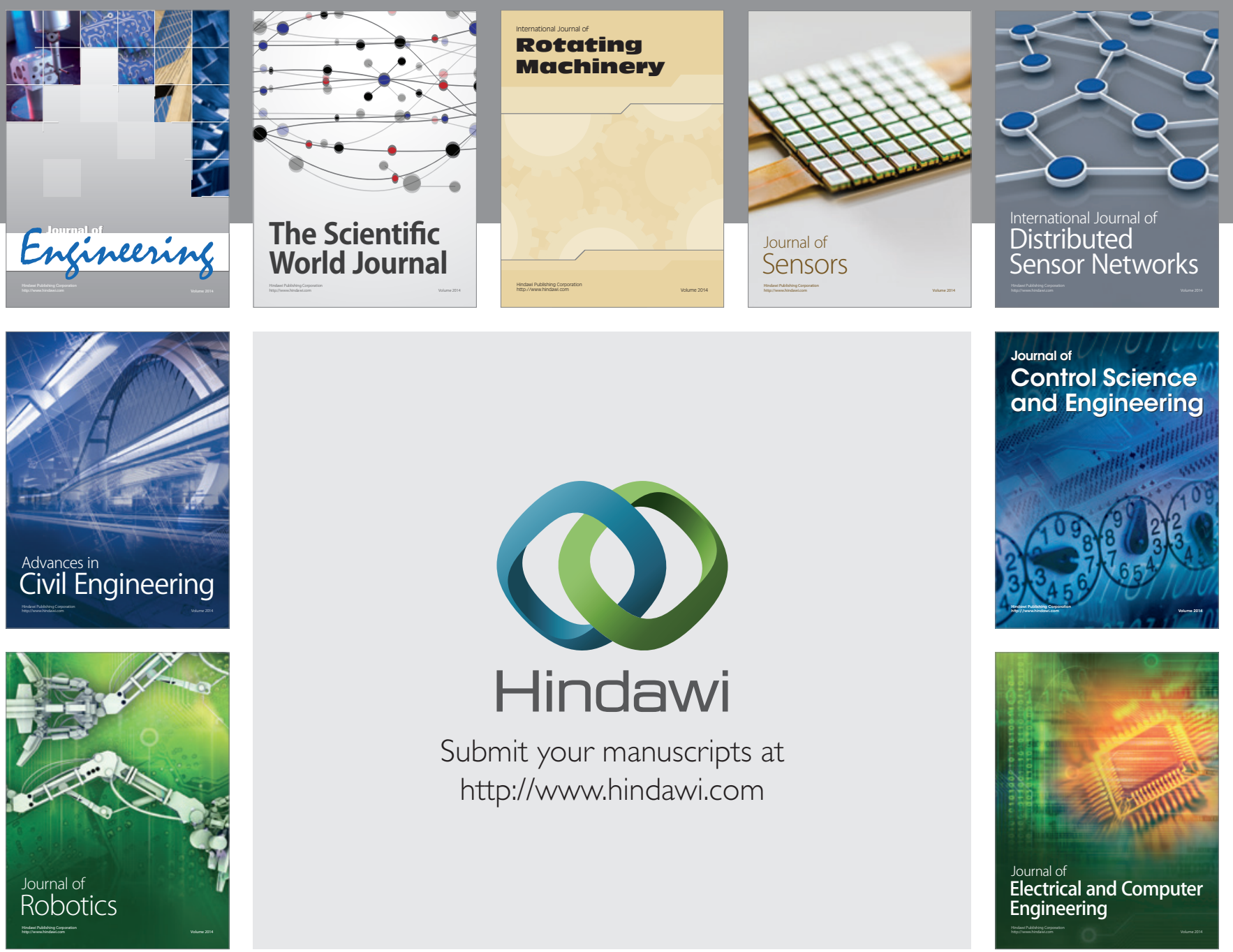

Submit your manuscripts at

http://www.hindawi.com
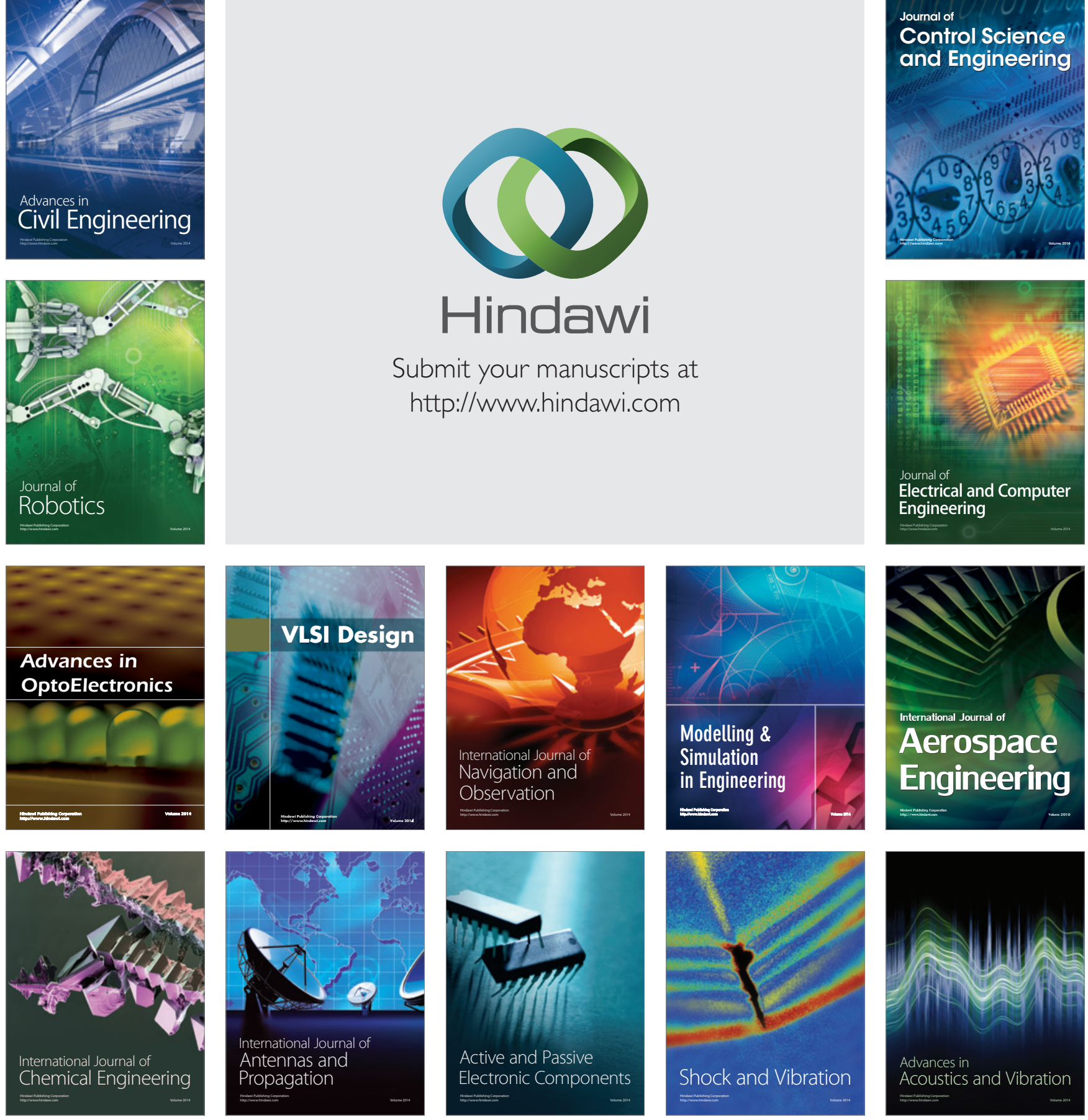